\title{
Togitare
}

\section{COMPARAÇÃO DA CARGA DE TRABALHO DE ENFERMAGEM ENTRE PACIENTES CLÍNICOS E CIRÚRGICOS EM TERAPIA INTENSIVA}

\author{
Ana Paula Aragão Santos ${ }^{1}$ (1) \\ Thialla Andrade Carvalho ${ }^{2}$ (1) \\ Juliana Prado Ribeiro Soares ${ }^{1}$ (1) \\ Paulo Roberto Lima Ferreira Coelho ${ }^{3}$ (c) \\ Eduesley Santana Santos ${ }^{1}$ (c)
}

\begin{abstract}
RESUMO
Objetivo: avaliar comparativamente a carga de trabalho de enfermagem entre pacientes clínicos e cirúrgicos em uma unidade de terapia intensiva.

Método: coorte prospectivo realizado em uma Unidade de Terapia Intensiva da capital sergipana, nordeste do Brasil, com os pacientes internados de agosto de 2018 a julho de 2019. Os dados foram analisados pelo software Epi-Info 7.2.

Resultados: os pacientes clínicos apresentaram maior tempo de internação e taxa de mortalidade. As médias, Simplified Acute Physiology Score 3, 24 horas e saída e Índice de Comorbidade de Charlson apresentaram diferença significativa entre os grupos. A média global da carga de trabalho segundo o Nursing Activities Score foi de 53,31 e observou-se diferença significativa em relação à carga de trabalho apenas na saída da unidade.

Conclusão: a aplicação dos índices e a mensuração da carga de trabalho entre os diferentes grupos, como neste estudo, auxilia no dimensionamento adequado, agrega segurança e qualidade assistencial.
\end{abstract}

DESCRITORES: Unidades de Terapia Intensiva; Carga de Trabalho; Cuidados de Enfermagem; Assistência ao Paciente; Cuidados Críticos.

\section{COMPARACIÓN DE LA CARGA DE TRABAJO DE ENFERMERÍA ENTRE PACIENTES CLÍNICOS Y QUIRÚRGICOS EN CUIDADOS INTENSIVOS}

\section{RESUMEN:}

Objetivo: evaluar comparativamente la carga de trabajo de enfermería entre pacientes clínicos y quirúrgicos en una unidad de cuidados intensivos. Método: cohorte prospectiva realizada en una Unidad de Cuidados Intensivos de la capital de Sergipe, noreste de Brasil, con pacientes ingresados desde agosto de 2018 hasta julio de 2019. Los datos se analizaron con el software Epi-Info 7.2. Resultados: los pacientes clínicos tuvieron una estancia hospitalaria más larga y una mayor tasa de mortalidad. Las medias, Simplified Acute Physiology Score 3, 24 horas y a la salida y el índice de Comorbilidad de Charlson mostraron diferencias significativas entre los grupos. La media global de la carga de trabajo según el Nursing Activities Score fue de 53,31 y se observó una diferencia significativa respecto a la carga de trabajo sólo en el momento del alta de la unidad. Conclusión: la aplicación de los índices y la medición de la carga de trabajo entre los diferentes grupos, como en este estudio, ayuda a dimensionar adecuadamente, añade seguridad y calidad asistencial.

DESCRIPTORES: Unidades de Cuidados Intensivos; Carga de Trabajo; Atención de Enfermería; Atención al Paciente; Cuidados Críticos. 
As medidas terapêuticas mais avançadas empregadas nas Unidades de Terapia Intensiva (UTI) permitiram reversão de doenças antes sem prognóstico. Estes avanços modificaram o perfil dos pacientes admitidos e, consequentemente, demandaram da equipe de enfermagem maior desenvoltura e segurança nos cuidados prestados ${ }^{(1)}$.

O cuidado de enfermagem livre de danos é um dos principais alvos para o alcance da qualidade da assistência e dos indicadores de saúde, uma vez que a ocorrência de evento adverso está diretamente relacionada com esse cuidado. $O$ dimensionamento inadequado destes profissionais, o excesso de tarefas assistenciais e o julgamento incorreto das necessidades dos pacientes constituem fatores intervenientes nesse processo(2). Ressalte-se, ainda, a relação entre o quantitativo de enfermagem e o aumento das taxas de complicações como infecções, quedas, pneumonia associada a ventilação mecânica, extubação acidental e lesões de pele - eventos que podem ser decisivos para o paciente em ambiente intensivo(1).

Para realizar o dimensionamento adequado e garantir a qualidade do cuidado prestado, o perfil de gravidade dos pacientes atendidos e as atividades de enfermagem necessárias à assistência devem ser consideradas ${ }^{(3)}$. O Nursing Activities Score (NAS) é um instrumento utilizado como possibilidade de sistematizar e gerenciar o cuidado com qualidade. Este instrumento tem sido associado ao uso de escores preditores de gravidade e mortalidade a fim de proporcionar condições para gerenciamento adequado, tendo em vista que a equipe superdimensionada é dispendiosa e uma equipe reduzida implica na redução da efetividade dos cuidados, o que prolonga a hospitalização, aumenta o risco de morbimortalidade e os custos para as instituições de saúde ${ }^{(4)}$.

O NAS é constituído por 23 itens com pontuações que variam do mínimo de 1,2 ao máximo de 32,0. $O$ resultado da soma das pontuações obtidas representa a porcentagem de tempo gasto nas últimas 24 horas pela equipe de enfermagem na assistência ao paciente, e sua pontuação final pode alcançar um máximo de $176,8 \%$. Cada ponto obtido no NAS equivale a 14,4 minutos $^{(6)}$.

Um estudo realizado na Holanda resultou em evidências importantes no que diz respeito à carga de trabalho de enfermagem. Foi observado que uma maior pontuação no escore de atividades de enfermagem por enfermeiro esteve associada a maior mortalidade hospitalar. Em contrapartida, não foi encontrada associação entre a proporção de pacientes por enfermeiro e a mortalidade. Deste modo, seria mais importante focar na carga de trabalho de enfermagem que os pacientes geram do que no número de pacientes que o enfermeiro deve cuidar na UTI(7).

Nesta perspectiva, sabe-se que a proposta do NAS é incluir a organização dos cuidados e o quantitativo de enfermagem de acordo com a especificidade e carga de trabalho que cada paciente gera. Assim, ao considerar que se pode atender a pacientes de origem distintas na UTI e que cada paciente se comporta subjetivamente, exigindo da enfermagem uma assistência diferenciada em termos qualitativos e quantitativos de assistência(8), questiona-se: qual a diferença da carga de trabalho de enfermagem entre pacientes clínicos e cirúrgicos no ambiente de terapia intensiva? Para responder à questão enunciada, o objetivo proposto foi avaliar comparativamente a carga de trabalho de enfermagem com pacientes cirúrgicos e clínicos em uma unidade de terapia intensiva de um hospital universitário. 
Trata-se de um coorte prospectivo realizado na unidade de terapia intensiva adulto de um hospital de ensino da capital sergipana, na região nordeste do Brasil. A UTI possui cinco leitos, conta com a equipe de enfermagem do turno matutino composta por quatro enfermeiros e oito técnicos de enfermagem, o turno da tarde três enfermeiros e nove técnicos, com carga horária de seis horas diárias. A equipe noturna tem um enfermeiro e cinco técnicos com plantão de 12 horas. Dos leitos, três são destinados aos pacientes cirúrgicos, um a pacientes das clínicas de internamento do hospital e um destinado ao Sistema Interfederativo de Garantia do Acesso Universal, sendo a redistribuição realizada para adequar-se a ocasionais demandas existentes.

A amostra do estudo foi composta por 169 pacientes, com idade maior ou igual a 18 anos, internados na UTI de agosto de 2018 a julho de 2019. A sistemática de coleta de dados ocorreu por meio do preenchimento do instrumento padronizado a partir dos dados dos prontuários. A coleta se iniciava a partir da admissão do paciente, sendo registrado diariamente nos sete primeiros dias e posteriormente no dia da alta ou óbito na UTI.

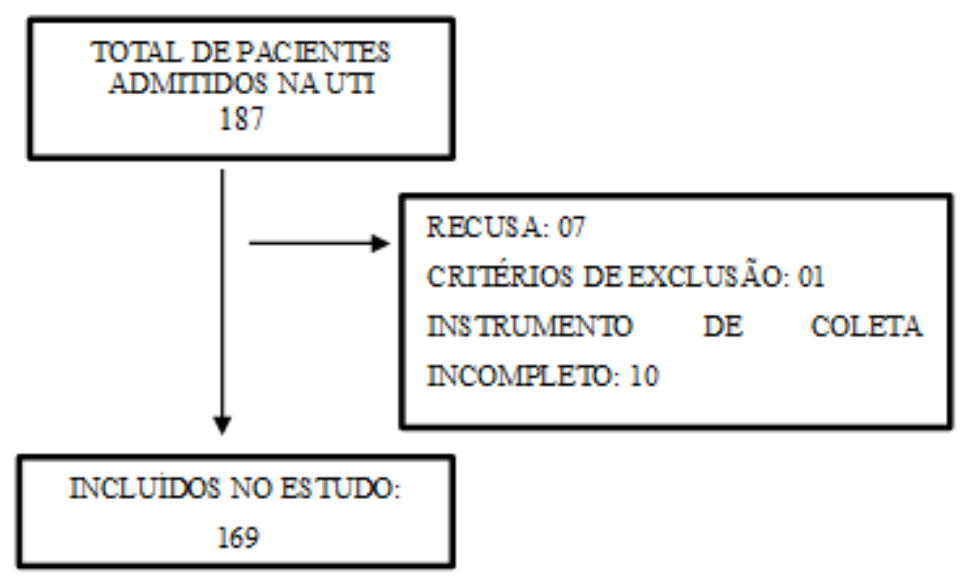

Figura 1 - Fluxograma de inclusão dos pacientes no estudo. Aracaju, SE, Brasil, 2020 Fonte: Autores (2020)

Participaram da coleta sete enfermeiros, três alunos de pós-graduação e um técnico de enfermagem, todos previamente treinados por um enfermeiro especialista com experiência clínica na área de UTI cardiológica.

O instrumento de coleta de dados foi subdividido em: dados sociodemográficos, caracterização clínica, suporte a admissão na UTI, valores laboratoriais e avaliação do índice de gravidade pelo Simplified Acute Physiology Score 3 (SAPS 3) e Índice de Comorbidade de Charlson (ICC). Além da avaliação da carga de trabalho de enfermagem pelo Nursing Activities Score (NAS) e desfechos.

A caracterização sociodemográfica e clínica da amostra foi realizada por meio da análise das variáveis: idade, sexo, setor de procedência e presença de comorbidades. Os diagnósticos foram agrupados de acordo com o sistema orgânico a partir do motivo da internação especificado em prontuário. Foi avaliada ainda a gravidade dos desfechos através do tempo de internação e mortalidade na UTI.

A carga de trabalho de enfermagem foi verificada utilizando o instrumento NAS(9). Conforme a publicação original, este foi desenvolvido como preditor de carga de trabalho de enfermagem, independente da gravidade do paciente, e consiste em um conjunto de 
itens relacionados às intervenções médicas e de enfermagem a que o paciente pode ser submetido na UTI; o resultado da soma das pontuações obtidas representa a porcentagem de tempo gasto em 24 horas.

O NAS é formulado em seis áreas do cuidado: suporte respiratório, cardiovascular, renal, neurológico, metabólico e atividades básicas, que é subdividido em oito categorias - monitorização e controles, investigações laboratoriais, medicação, procedimentos de higiene, cuidados com dreno, mobilização e posicionamento, suporte e cuidado aos familiares e pacientes e tarefas administrativas e gerenciais ${ }^{(5)}$. O NAS era calculado após 24 horas da admissão e diariamente até o sétimo dia, tendo como base as informações das últimas 24 horas disponíveis em prontuário. Posteriormente, era calculado novamente no dia do óbito ou alta da UTI, baseado nas intervenções até o horário da saída, independente de completarem ou não 24 horas.

O ICC é um sistema de classificação de gravidade que utiliza dados de registro de diagnósticos secundários para atribuir o peso da morbidade. $O$ método utiliza a pontuação de acordo com as comorbidades apresentadas pelo paciente; a soma total é acrescida de uma pontuação de acordo com a idade, a partir dos 50 anos acrescentando-se um ponto a cada 10 anos $^{(10)}$. O ICC era calculado na admissão do paciente na UTI, com base nas informações disponibilizadas em prontuário ou colhidas com o paciente ou familiar responsável.

O SAPS 3 é um escore de gravidade, cujo valor mais elevado indica que maior é a gravidade esperada. Além disso, o valor desse índice pode ser traduzido em probabilidade de morte dos pacientes. Este escore avalia variáveis demográficas, razões pela admissão na UTI e variáveis fisiológicas que representam o grau de comprometimento da doença e avaliação do estado de saúde prévio à admissão hospitalar, indicadora da condição prémórbida ${ }^{(11)}$. O SAPS 3 era calculado no momento da admissão e posteriormente na data da alta ou óbito na UTI.

Os dados foram analisados no software Epi-Info, versão 7.2. Para as variáveis categóricas, foram calculadas as frequências de distribuição; para as variáveis quantitativas, as medidas de tendência central e de dispersão correspondentes. A distribuição da normalidade dos dados foi testada pelo teste de D'Agostino-Pearson. As diferenças entre os grupos clínicos e cirúrgicos foram verificadas para as variáveis categóricas pelo teste qui-quadrado; para as variáveis quantitativas utilizou-se o teste de T ou Mann-Whitney.

A pesquisa respeitou os preceitos éticos conforme resolução 466/12 do Conselho Nacional de Saúde, sendo aprovada pelo comitê de ética em pesquisa da Universidade Federal de Sergipe, através do parecer $n^{\circ} 2.830 .187$. Faz parte do projeto de pesquisa intitulado "Caracterização clínica, perfil de gravidade e desfecho de pacientes em Unidades de Terapia Intensiva".

\section{RESULTADOS}

Dos 169 pacientes incluídos no estudo, a maioria, 120 (71,01\%), foi classificada como sendo cirúrgicos. Não houve diferença significativa quanto ao sexo. Os pacientes clínicos apresentaram maior tempo de permanência na UTI, assim como maior taxa de mortalidade. As médias SAPS 324 horas e saída e ICC em pacientes cirúrgicos e clínicos apresentaram diferença estatisticamente significativa (Tabela 1). 
Tabela 1 - Características clínicas e demográficas de pacientes críticos internados em UTI segundo tipo de internação, de agosto de 2018 a julho de 2019. Aracaju, SE, Brasil, 2020

\begin{tabular}{|c|c|c|c|c|}
\hline & Clínico & Cirúrgico & Total & p-valor \\
\hline Total & $28,99(49)$ & $71,01(120)$ & 100 (169) & \\
\hline \multicolumn{5}{|l|}{ Sexo, \% (n) } \\
\hline Masculino & $44,90(22)$ & $44,05(52)$ & $43,79(74)$ & \multirow{2}{*}{$0,493^{*}$} \\
\hline Feminino & $55,10(27)$ & $55,95(68)$ & $56,21(95)$ & \\
\hline \multicolumn{5}{|l|}{ Condições de saída \% (n) } \\
\hline Sobreviventes & $38,78(19)$ & $92,50(111)$ & $76,92(130)$ & \multirow{2}{*}{$<0,001 *$} \\
\hline Não sobreviventes & $61,22(30)$ & $7,50(9)$ & $23,08(39)$ & \\
\hline Idade - média (DP) & $55,59(16.93)$ & $56,27(16.29)$ & $56.07(16,43)$ & $0,809 * \star \star$ \\
\hline Tempo de permanência mediana (IIQ) & $8(4-13)$ & $1(1-3)$ & $2(1-6)$ & $<0,001 * *$ \\
\hline SAPS $324 \mathrm{~h}$ - média (DP) & $29,06(10,57)$ & $15,47(9,13)$ & $19,43(11,38)$ & $<0,001 * \star \star$ \\
\hline SAPS 3 saída - média (DP) & $27,22(14,22)$ & $12,62(9,80)$ & $16,86(13,03)$ & $<0,001 * * *$ \\
\hline ICC admissão - média (DP) & $4,41(2,88)$ & $3,11(2,83)$ & $3,49(2,90)$ & $<0,001 * * *$ \\
\hline \multicolumn{5}{|c|}{$\begin{array}{l}\text { Legenda: SAPS } 3 \text { - Simplified Acute Physiology Score } 3 \text { (escore fisiológico agudo simplificado); ICC - Índice de Comorbidade de } \\
\text { Charlson; Med - Mediana; IIQ- intervalo interquart; DP - Desvio Padrão Nota: }{ }^{\star} \text { Nível descritivo de probabilidade do teste Qui- } \\
\text { quadrado; }{ }^{\star \star} \text { Nível descritivo de probabilidade do teste Mann-Whitney; }{ }^{\star \star * N i v e l ~ d e s c r i t i v o ~ d e ~ p r o b a b i l i d a d e ~ d o ~ T e s t e ~ T ~} \\
\text { Fonte: Autores (2020) }\end{array}$} \\
\hline
\end{tabular}

Na amostra total, a média da carga de trabalho de enfermagem segundo o NAS foi de 53,31 , não houve diferença estatística significativa entre a média dos dois grupos. Contudo, observou-se diferença significativa em relação à carga de trabalho de enfermagem na saída da UTI, sendo que o maior valor do escore NAS correspondia aos pacientes clínicos (Tabela 2).

Tabela 2 - Média do Nursing Activities Score (NAS), segundo tipo de internação dos pacientes internados em UTI, de agosto de 2018 a julho de 2019. Aracaju, SE, Brasil, 2020

\begin{tabular}{lcccc} 
& $\begin{array}{c}\text { Clínico } \\
\text { Média (DP) }\end{array}$ & $\begin{array}{c}\text { Cirúrgico } \\
\text { Média (DP) }\end{array}$ & $\begin{array}{c}\text { Total } \\
\text { Média (DP) }\end{array}$ & p-valor \\
\hline NAS médio & $54,39(6,36)$ & $52,87(8,05)$ & $53,31(7,61)$ & 0,195 \\
\hline NAS 24h & $50,32(5,10)$ & $48,66(7,92)$ & $49,44(6,75)$ & 0,227 \\
\hline NAS saída & $65,22(13,09)$ & $55,41(11,08)$ & $58,26(12,48)$ & $<0,001$ \\
\hline NAS 24h sobreviventes & $50,44(4,35)$ & $48,21(8,41)$ & $48,87(7,47)$ & 0,29 \\
\hline NAS 24h não sobreviventes & $50,24(5,61)$ & $51,06(4.00)$ & $50,42(5,28)$ & 0,702 \\
\hline NAS saída sobreviventes & $58,17(10,40)$ & $54,08(9,30)$ & $54,67(9,54)$ & 0,086 \\
\hline NAS saída não sobreviventes & $69,71(12,75)$ & $71,88(17,55)$ & $70,21(13,78)$ & 0,684
\end{tabular}

Legenda: NAS - Nursing Activities Score; DP-Desvio Padrão.

Fonte: Autores (2020) 
O estudo apresenta a comparação da carga de trabalho de enfermagem medida pelo NAS entre os pacientes clínicos e cirúrgicos internados em uma UTI geral de um hospital de ensino. Observou-se não haver diferença significante quanto ao sexo, diferindo da maioria dos estudos, cujos maiores percentuais são masculinos ${ }^{(12-15)}$. Contudo, é compatível com o revelado por estudo da Associação de Medicina Intensiva Brasileira, que apresenta um percentual de internações, semelhante ao encontrado nesta casuística, com 48,24\% mulheres e $51,76 \%$ homens no ano de 2019 nas UTI adulto do Brasil(16).

Assim, a diferença numérica, embora pequena, encontrada neste estudo pode ser justificada por tratar-se de um hospital com encaminhamentos eletivos e em virtude da procura relativamente baixa dos homens ao serviço de saúde, que leva ao aumento da gravidade da condição e consequentemente são mais frequentes nos hospitais porta aberta e que atendem urgências ${ }^{(17)}$.

A idade média global dos pacientes atendidos foi semelhante ao encontrado na literatura, variando entre 56,4 e 77 anos $^{(1,18)}$, esta característica segue um padrão decorrente da mudança demográfica no país, cuja população de idosos tem aumentado progressivamente, modificando o perfil dos pacientes atendidos e elevando o número de idosos no ambiente intensivo(18).

O fato de os pacientes clínicos apresentarem maior tempo de permanência na UTI é explicado pelo esperado perfil de descompensação, menor nível de funcionalidade, com fator causal que, na maioria das vezes, requer maior tempo para resolutividade. Além do peso das comorbidades que, como observado, os paciente clínicos demonstraram maior média no ICC, correlacionando a maior tempo de internação na UTI e maior risco de mortalidade intra-hospitalar ${ }^{(19)}$, tendo em vista que este é um importante identificador da reserva orgânica, revelando o impacto na sobrevida destes indivíduos no ambiente crítico.

Além disso, a carga da doença mensurada pelo ICC pode não ser indicador apenas de maior gravidade, mas também da possibilidade de prováveis complicações, uma vez que o paciente pode apresentar maior dificuldade de recuperação, sendo esta acrescida se o paciente for idoso(19).

Já a procedência cirúrgica também compusera a maioria dos internados no ambiente intensivo ${ }^{(8,12-14)}$. O número elevado de pacientes internados no pós-operatório decorre do fato que algumas cirurgias têm como protocolo a vinda do paciente para UTI, pois se exige atenção na evolução, uma vez que complicações são ainda frequentes, ocorrendo em cerca de um terço dos pacientes. A idade, SAPS 3 e SOFA na admissão da UTI estão associados à mortalidade em 28 dias e complicações pós-operatórias ${ }^{(13)}$.

Apesar do perfil cirúrgico eletivo, outro fator que pode influenciar na necessidade de monitorização rigorosa, e consequentemente internação na UTI, é o fato de que o hospital é referência em cirurgias eletivas do aparelho digestivo, cabeça e pescoço, torácica, ginecológica e mastologia cirúrgica, relacionadas ao diagnóstico, ressecção, reconstrução e paliação, sendo a neoplasia o diagnóstico mais prevalente para a internação na UTI.

Contudo, apesar da prevalência da procedência cirúrgica, a média dos valores do SAPS 3 de 24 horas dos pacientes cirúrgicos foi significativamente menor que a encontrada em um estudo com pacientes cirúrgicos de duas UTI de hospitais terciários da região de São

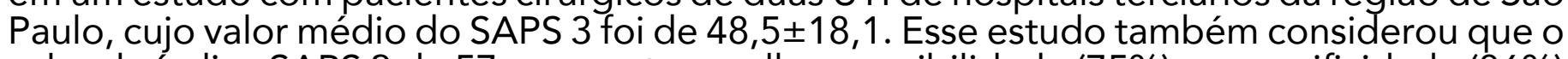
valor do índice SAPS 3 de 57 apresentou melhor sensibilidade (75\%) e especificidade (86\%) para mortalidade hospitalar ${ }^{(11)}$. Logo, escore menor ou igual a 57 apresentam maiores taxas de sobrevivência, o que é visto neste estudo, considerando a baixa taxa de mortalidade $(7,50 \%)$ dos pacientes cirúrgicos. 
Quanto à carga de trabalho de enfermagem, o NAS médio global foi consideravelmente menor que o encontrado em outras investigações em que o NAS variou de 61,9 a $98,37^{(12-}$ 15,18). Não houve diferença estatística significativa em NAS médio e de 24 horas entre os grupos, ou seja, os valores do NAS foram semelhantes para pacientes cirúrgicos e clínicos, tanto na média geral quanto na admissão.

Mesmo com um perfil de cirúrgicos eletivos, o pós-operatório demanda dos profissionais tanto atividades assistenciais diretas quanto organizacionais e administrativas. Além disso, por se tratar de pós-operatório imediato, estes pacientes requerem atenção às possíveis complicações e cuidados decorrentes de cada especificidade cirúrgica.

Portanto, observou-se que, embora os pacientes fossem cirúrgicos eletivos e permanecessem pouco tempo na UTI, eles demandaram da equipe de enfermagem tanto quanto os pacientes clínicos. Esta afirmação corrobora com o encontrado em estudo, que evidenciou uma correlação negativa $(-0,23)$ entre o NAS e tempo de internação, ou seja, quanto menor o tempo de internação, maior o $\operatorname{NAS}^{(8)}$.

Nas investigações, o NAS da admissão obtivera valor maior, diminuindo ao longo da internação nos sobreviventes e aumentando progressivamente nos não sobreviventes ${ }^{(12)}$. Neste estudo, o valor médio do NAS 24 horas e saída se manteve linear nos pacientes sobreviventes e aumentou nos não sobreviventes. Pesquisa realizada em um hospital de ensino evidenciou valor médio do NAS maior tanto para pacientes clínicos NAS $66,7 \%$, quanto cirúrgicos NAS $72,5 \%{ }^{(8)}$.

O NAS da saída apresentou diferença estatística significativa, sendo a maior média referente ao grupo clínico. Também observou-se uma diferença significativa entre os valores de SAPS 3 de saída, evidenciando um perfil de maior gravidade dos pacientes clínicos, consequentemente demandando da equipe de enfermagem a implementação de intervenções direcionadas e avaliação sistemática. Além disso, o desfecho mortalidade no momento da saída requer da equipe uma série de atividades administrativas e de suporte familiar, também levados em consideração na aplicação do NAS. Portanto, a diferença encontrada no NAS de saída correlaciona-se com a taxa de mortalidade $(23,08 \%)$ dos pacientes clínicos. Esta associação é observada também em outros estudos, cuja mortalidade também elevou o NAS de saída ${ }^{(8,12)}$.

A taxa de mortalidade evidenciada encontra-se entre os números descritos em estudos nacionais, variando entre 10,3 e 36,6 $6^{(11,15,20)}$, porém, o valor médio do SAPS 3 mostrou-se menor em comparação aos estudos realizados em UTI da região de São Paulo, com médias do SAPS 3 em 44,2 a 48,5(11,14). Vale ressaltar que, apesar do valor dos escores de gravidade dos pacientes serem menores, a taxa de mortalidade permaneceu semelhante.

Consoante ao encontrado em outro estudo(12), a UTI em questão possui um número pequeno de leitos disponíveis, assim como o estado, retardando intervenções que previnem a deterioração do quadro clínico e melhora do prognóstico do paciente, pois muitos são transferidos de hospitais regionais dos municípios circunvizinhos e do interior do estado. Este fato pode influenciar nos valores do NAS sem queda ao longo da internação e na taxa de mortalidade.

Desta forma, o NAS enquanto instrumento que possibilita a análise da carga de trabalho de enfermagem na UTI, leva em consideração os diversos fatores e especificidades dos pacientes atendidos. O seu uso em estudos é bem difundido, com populações críticas de diferentes conformações, evidenciando sua viabilidade para mensurar a carga de trabalho de enfermagem em diferentes grupos de pacientes ${ }^{(14)}$. A mensuração da carga de trabalho considerando as admissões clínicas e cirúrgicas e a demanda de cuidado a estes pacientes é uma ferramenta útil ao enfermeiro no seu dia a dia, podendo agregar maior segurança e qualidade assistencial.

Como limitação desta pesquisa, ressalta-se a utilização dos dados de uma única instituição e a diferença entre o quantitativo de pacientes entre os grupos. Contudo, os 
resultados sugerem a viabilidade da metodologia utilizada, além de revelarem informações importantes a respeito da carga de trabalho de enfermagem para pacientes clínicos e cirúrgicos.

\section{CONCLUSÃO}

Os resultados permitiram concluir que, independente do tempo de internação e procedência, os pacientes cirúrgicos e clínicos apresentaram carga de trabalho de enfermagem estatisticamente semelhante. $O$ valor médio global do NAS encontrado foi menor que o descrito em diversos estudos, contudo, a UTI possui uma alta taxa de mortalidade dos pacientes clínicos, elevando a carga de trabalho de enfermagem durante a saída destes pacientes.

Assim, pondera-se que a aplicação de índices e a mensuração da carga de trabalho comparativamente, tais como os utilizados nesse estudo, pode auxiliar os gerentes de enfermagem das unidades intensivas a dimensionarem adequadamente, visando maior segurança e qualidade assistencial, além de possibilitar a apresentação dos resultados de forma prática e objetiva.

\section{REFERÊNCIAS}

1. Cargnin MC dos S, Ottobelli C, Barlem ELD, Cezar-Vaz MR. Tecnologia no cuidado da enfermagem e a carga de trabalho em UTI. Rev enferm UFPE on line. [Internet]. 2016 [acesso em 29 nov 2020] 10(2). Disponível em: https://periodicos.ufpe.br/revistas/revistaenfermagem/article/view/11035.

2. Toffoletto MC, Oliveira EM de, Andolhe R, Barbosa RL, Padilha KG. Comparison between patients everity and nursing worload before and after the occurrence of adverse events in elderly in critical care. Texto contexto - enferm. [Internet]. 2018 [acesso em 29 jul 2019]; 27(1). Disponível em: http://dx.doi. org/10.1590/0104-070720180003780016.

3. Vandresen L, Pires DEP de, Lorenzetti J, Andrade SR de. Classificação de pacientes e dimensionamento de profissionais de enfermagem: contribuições de uma tecnologia de gestão. Rev Gaúcha Enferm. 2018 [acesso em 29 nov 2020]; 39. Disponível em: https://doi.org/10.1590/1983-1447.2018.2017-0107.

4. Castro MCN e, Dell'Acqua MCQ, Unger IC, Cyrino CMS, Almeida PMV de. Severity and workload of nursing with patients seeking admission to an intensive care unit. Esc. Anna Nery [Internet]. 2018 [acesso em 29 jul 2019]; 22(1). Disponível em: https://doi.org/10.1590/2177-9465-ean-2017-0167.

5. Queijo AF, Padilha KG. Nursing Activities Score (NAS): cross-cultural adaptation and validation to Portuguese language. Rev. esc. enferm. USP [Internet]. 2009 [acesso em 01 out 2019]; 43. Disponível em: https://doi.org/10.1590/S0080-62342009000500004.

6. Cardoso RB, Caldas CP, Souza PA de. Nursing activities score e sua correlação com a teoria do conforto de kolcaba: reflexão teórica. Enferm. Foco [Internet]. 2019 [acesso em 05 jan 2020]; 10(1). Disponível em: https://doi.org/10.21675/2357-707X.2019.v10.n1.1347.

7. Margadant C, Wortel S, Hoogendoorn M, Bosman R, Spijkstra JJ, Brinkman S, et al. The Nursingc Activities Score Per Nurse Ratio Is Associated With In-Hospital Mortality, Whereas the Patients Per Nurse Ratio Is Not*. Crit. Care Med. 2020 [acesso em 25 fev 2020]; 48(1). Disponível em: http://doi.org/10.1097/ CCM.0000000000004005. 
8. Romano JL, Garcia PC, Silva DV, Moura BRS, Nogueira L de S. Type of admission and nursing workload of critical patients: a cross-sectional study. Nurs Crit Care. [Internet]. 2019 [acesso em 29 nov 2020]; 24(6). Disponível em: https://doi.org/10.1111/nicc.12408.

9. Miranda DR, Nap R, Rijk A de, Schaufeli W, lapichino G. Nursing activities score. Crit Care Med. [Internet]. 2003 [acesso em 26 dez 2019]; 31(2). Disponível em: https://www.ncbi.nlm.nih.gov/ pubmed/12576939.

10. Martins M, Blais R, Miranda NN de. Avaliação do índice de comorbidade de Charlson em internações da região de Ribeirão Preto, São Paulo, Brasil. Cad. Saúde Pública [Internet]. 2008 [acesso em 19 nov 2019]; 24(3). Disponível em: https://doi.org/10.1590/S0102-311X2008000300018.

11. Silva Junior JM, Malbouisson LMS, Nuevo HL, Barbosa LGT, Marubayashi LY, Teixeira IC, et al. Applicability of the Simplified Acute Physiology Score (SAPS 3) in Brazilian Hospitals. Rev. Bras. Anestesiol. [Internet]. 2010 [acesso em 12 nov 2019]; 60(1). Disponível em: https://doi.org/10.1590/ S0034-70942010000100003.

12. Nogueira L de S, Koike KM, Sardinha DS, Padilha KG, Sousa RMC de. Nursing workload in public and private intensive care units. Rev. Bras. Ter. Intensiva [Internet]. 2013 [acesso em 28 dez 2019]; 25(3). Disponível em: https://doi.org/10.5935/0103-507X.20130039.

13. Silva Júnior JM, Chaves RC de F, Corrêa TD, Assunção MSC de, Katayama HT, Bosso FE, et al. Epidemiologia e desfecho dos pacientes de alto risco cirúrgico admitidos em unidades de terapia intensiva no Brasil. Rev. bras. ter. intensiva [Internet]. 2020 [acesso em 29 nov 2020]; 32(1). Disponível em: https://doi.org/10.5935/0103-507x.20200005.

14. Goulart LL, Carrara FSA, Zanei SSV, Whitaker IY. Nursing workload related to the body mass index of critical patients. Acta paul. enferm. [Internet]. 2017 [acesso em 28 dez 2019]; 30(1). Disponível em: https:// doi.org/10.1590/1982-0194201700006.

15. Silva BL da, Gaedke MÂ. Nursing activites score: avaliando a carga de trabalho de enfermagem no cuidado intensivo. Rev. Enferm. Atual In Derme. [Internet] 2019 [acesso em 28 dez 2019]; 89(27). Disponível em: https://revistaenfermagematual.com/index.php/revista/article/view/380.

16. Associação de Medicina Intensiva Brasileira (AMIB). Registro Nacional de Terapia intensiva. [Internet] 2019. [acesso em 17 jan 2020]. Disponível em: http://www.utisbrasileiras.com.br/uti-adulto/caracteristicasdemograficas/.

17. Rodriguez AH, Bub MBC, Perão OF, Zandonadi G, Rodriguez M de JH. Características epidemiológicas e causas de óbitos em pacientes internados em terapia intensiva. Rev. Bras. Enferm. [Internet]. 2016 [acesso em 29 nov 2020]; 69(2). Disponível em: https://doi.org/10.1590/00347167.2016690204i.

18. Vieira AM, Parente EA, Oliveira L de S, Queiroz AL, Bezerra ISAM, Rocha HAL. Características de óbitos dos pacientes internados em uma unidade de terapia intensiva de hospital terciário. J Health Biol Sci. [Internet]. 2019 [acesso em 17 jan 2020]; 7(1). Disponível em: http://docs.bvsalud.org/ biblioref/2019/01/969718/5-1999.pdf.

19. Ferretti-Rebustini RE de L, Bispo N da S, Alves W da S, Dias TN, Santoro CM, Padilha KG. Level of acuity, severity and intensity of care of adults and older adults admitted to the Intensive Care Unit. Rev. esc. enferm. USP [Internet]. 2019 [acesso em 08 mar 2020]; 53. Disponível em: https://doi.org/10.1590/ s1980-220x2017051403416.

20. Miranda GMD, Mendes A da CG, Silva ALA da. Population aging in Brazil: current and future social challenges and consequences. Rev. bras. geriatr. gerontol. [Internet]. 2016 [acesso em 28 dez 2019]; 19(3). Disponível em: http://dx.doi.org/10.1590/1809-98232016019.150140. 
COMO REFERENCIAR ESTE ARTIGO:

Santos APA, Carvalho TA, Soares JPR, Coelho PRLF, Santos ES. Comparação da carga de trabalho de enfermagem entre pacientes clínicos e cirúrgicos em terapia intensiva. Cogitare enferm. [Internet]. 2021 [acesso em "colocar data de acesso, dia, mês abreviado e ano"]; 26. Disponível em: http://dx.doi.org/10.5380/ce.v26i0.73689.

Recebido em: 13/05/2020

Aprovado em: 06/12/2020

Editora associada: Cremilde Aparecida Trindade Radovanovic

Autor Correspondente:

Ana Paula Aragão Santos

Universidade Federal de Sergipe - Aracaju, SE, Brasil

E-mail: enfanapaulaa@gmail.com

Contribuição dos autores:

Contribuições substanciais para a concepção ou desenho do estudo; ou a aquisição, análise ou interpretação de dados do estudo - APAS, TAC, JPRS, PRLFC, ESS

Elaboração e revisão crítica do conteúdo intelectual do estudo - APAS, TAC, JPRS, ESS

Aprovação da versão final do estudo a ser publicado - APAS, TAC, JPRS, PRLFC, ESS

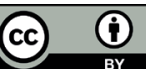

Copyright $\odot 2021$ Este é um artigo em acesso aberto distribuído nos termos da Licença Creative Commons Atribuição, que permite o uso irrestrito, a distribuição e reprodução em qualquer meio desde que o artigo original seja devidamente citado. 